\title{
CLUSTER HEAD SELECTION USING RF SIGNAL STRENGTH
}

\author{
Scott Fazackerley, Alan Paeth, Ramon Lawrence* \\ University of British Columbia Okanagan \\ scott.fazackerley@alumni.ubc.ca, alan.paeth@ubc.ca, ramon.lawrence@ubc.ca
}

\begin{abstract}
The LEACH algorithm for selecting cluster heads is a probabilistic method which produces clusters with a large variation of link distances and uneven energy consumption during the data transmission phase. To address this issue, a RF signal strength algorithm based on link quality is presented. Using a competitive distributed algorithm, nodes attempt to reduce the overall energy required for transmission in addition to forming favourable clusters based on Received Signal Strength Indication (RSSI) density and quality. Cluster heads form in areas of high node density leading to a significant reduction in transmission link length, a reduced variance in link length distribution and greater opportunity for energy savings through data aggregation. Simulations show that cluster heads selected by this algorithm form clusters with a lower average link length and have less link distance variability. This produces a lower and more evenly distributed energy cost per node in the network.
\end{abstract}

Index Terms - Wireless Sensor Networks, Cluster Head Selection, Link Length, Energy Efficiency, RSSI

\section{INTRODUCTION}

Cluster head selection is an important problem in sensor networks. Cluster-based routing has been shown to be more energy efficient and increase the network lifetime through data aggregation. The goal is to select cluster heads that minimize transmission costs and energy usage. Various algorithms have been proposed including probabilistic approaches such as LEACH [1], Max-Min [2], HEED [3], graph properties [4], and genetic algorithms [5].

In this work we propose a cluster head selection algorithm that uses RF signal strength for head selection. The 802.15.4 standard [6] supports the determination of the signal strength of neighbouring nodes. This is implemented in many commercial radios. A cluster head is selected based on its perceived RF signal strength of its neighbours. This approach has several advantages. First, unlike relying on Euclidean distance which requires localization or network knowledge,

\footnotetext{
*The authors are funded by NSERC CGS, a NSERC Discovery grant, and the Irving K. Barber Endowment Fund.
}

using RF signal strength compensates for the network properties. Nodes close by distance may not be close by signal requiring more power for transmissions. This also compensates for dead spots, uneven propagation, and changing RF propagation characteristics. Additionally, cluster heads located in areas of higher node density are expected to have a lower average distance between end nodes and the cluster head [7], leading to a greater probability of data aggregation.

The contributions of this paper are:

- A cluster head selection algorithm based on RF signal strength.

- Simulation comparisons with LEACH that demonstrate the algorithm's improved performance and lower energy usage.

The paper outline is as follows. In Section 2 we overview previous cluster head selection approaches and discuss the 802.15.4 standard support for RF signal strength. Our cluster head selection algorithm is in Section 3. Experimental simulation results are in Section 4. The paper closes with future work and conclusions.

\section{PREVIOUS WORK}

Cluster-based routing is often more energy efficient in sensor networks than planar routing [8]. Cluster-based routing requires the formation of clusters, and the selection of cluster heads to communicate with end nodes in the cluster and the base station (also called the sink).

Seminal work in the area of cluster head selection is the LEACH protocol. LEACH [1] is a probabilistic protocol that selects a cluster head by random waiting. LEACH-C [1] is a variation that uses a centralized clustering algorithm at the sink based on simulated annealing to determine $k$ optimal clusters. This is an NP hard problem [9]. HEED [3] is a probabilistic method that determines the probability using the residual energy of each node. Other algorithms use graph properties such as node degree [4] which seeks to optimize the number of clusters based on hexagonal closet packing, or the allowed number of hops from a cluster head candidate [2]. Many protocols consider cluster head rotation as the cluster head will consume power quicker than end devices. Our focus 
will be only on the selection with the understanding that rotation can be accomplished by periodically applying the head selection algorithm.

There are some challenges in these approaches. The randomness in LEACH does not provide any optimal guarantees [1] and may encounter rounds of communication where $k$ cluster heads are not available. LEACH assumes that all nodes in the topology can intercommunicate, but this is impractical for large scale deployments were the network may encounter the hidden terminal problem. LEACH-C assumes that all nodes can communicate with the sink which is not practical. Extensive work has been done to extend the cluster head selection algorithm in LEACH [10, 11, 12]. These protocols focus on energy consumption of a single node, but do not consider the cost of transmission in cluster head selection. The degree based protocols assume prior knowledge of the graph topology which is often not feasible with field deployment [13] and is not practical for mobile or dynamic network topologies. The convergent nature of Min-Max [2] also suffers from node abandonment.

There has been no cluster head selection protocol that is based on link quality indicator (LQI) for received packets. This is surprising given the built-in support for RF signal strength capture in 802.15.4 [6, 14] and its implementation in sensor network devices. Using RF signal strength is responsive to environmental characteristics and does not require localization or knowledge of the graph topology to be used.

\section{CLUSTER HEAD SELECTION BY RF SIGNAL STRENGTH}

The RSSI clustering algorithm is based on the concept that a node operates with two distinct goals. As an end node, each device attempts to optimize its own energy consumption by associating with cluster heads that require the smallest amount of transmission energy. Additionally, nodes will not associate with cluster heads if the required link energy falls below a specific threshold. When a device operates as a cluster head, it attempts to optimize the number of devices that associate with it, which increases the chances for data aggregation. Devices that are in dense clusters will become cluster heads faster than devices in sparse areas of the network. Our approach is as follows:

Step 1: In the initial phase, each node starts in a nonassociated state. Each node runs an active scan which searches for active clusters. If a cluster head is found, the node will evaluate the RSSI of the response from each cluster head and determine if the strength of signal is above the required threshold, $R S S I_{\text {thres }}$. If the value is above the required threshold, the node will enter into association with the cluster head. If multiple cluster heads are available, the node will associate with the device that presents the strongest RSSI.

Step 2: If no suitable cluster head is located, the node enters into the topology discovery phase. Each non-associated node broadcasts a node discovery request to the network with a time-out value. The time-out value is the amount of time allowed for response to the message. Non-associated nodes that receive the request will respond with their configuration using a random hold off time based on the time-out value. All responses received after the time-out expires are ignored.

Step 3: Once the response to the discovery request has been received, each node orders the received RSSI values in decreasing order and computes its promotion factor $P_{\text {fact }}$. The promotion factor determines the likely hood of a device promoting itself to become a cluster head. The promotion factor is determined as:

$$
P_{\text {fact }}= \begin{cases}\frac{e^{n} \sum_{i=1}^{n} R S S I_{i}}{n} & \text { if } n>0 \\ \infty & \text { if } n=0\end{cases}
$$

where $n$ is the number of responses received, and $R S S I_{i}$ is the $i$-th strongest received signal strength from a responding node. The number of responses, $n$, is limited by the expected maximum number of responses from nodes in a dense network, based on available network bandwidth, for the selected timeout period.

Step 4: Each device enters a cluster head promotion phase, where it will wait $x$ number of cycles before attempting to promote itself to a cluster head and associate nodes with itself. The number of cycles $x=\frac{C}{P_{\text {fact }}}$ where $C$ is platform and network dependent (depends on the processor speed). The larger the value of the promotion factor, the sooner a device will promote itself. In the case where $P_{\text {fact }}=\infty$, no other nonassociated nodes are visible, so the node immediately promotes itself to be a cluster head.

Step 5: During the cluster head promotion phase, each node engages in periodic active scans where it listens for beacon announcements from new cluster heads. If a cluster head is found before the node promotes itself, it evaluates the RSSI of the cluster head announcement message against $R S S I_{\text {thres }}$. If the signal strength is above the threshold, it terminates the cluster head promotion algorithm and sends an association request to the cluster head. If multiple cluster heads are located that satisfy the association conditions, the node associates with the device with the strongest RSSI.

Step 6: Once a node has been associated with a cluster head or the cluster head promotion algorithm has completed, each device enters the optimization phase. During the optimization phase, each device continues to run periodic active scans, searching for cluster heads. Each device strives to minimize the transmission path cost to its cluster head in an effort to maximize lifetime. If a cluster head is located that has a higher RSSI than the current association and is above $R S S I_{\text {thres }}$, the node will break the association with its current cluster head and send an association request to the new cluster head. 
At anytime during the algorithm, if a cluster head dies or a device loses association with a cluster head it will continue to scan for available cluster heads and re-start the cluster head promotion phase to determine a new promotion factor for itself. Note that the number of cluster heads is dictated by $R S S I_{\text {thres }}$ whereas in LEACH, $k$ is explicitly defined. A larger value of $R S S I_{\text {thres }}$ results in more cluster heads.

\section{EXPERIMENTAL RESULTS}

To test the approach, we built a simulator written in the Java language to compare the clustering algorithms. The parameters of the simulator were number of nodes, $\mathrm{N}=100$, over a $100 \mathrm{~m} \mathrm{x} 100 \mathrm{~m}$ grid. Note that $k=4$ (four cluster heads) is suggested by [1] to be an optimal number of cluster heads for this model. The timeout value is 2.5 seconds. All nodes are able to transmit and receive across the entire grid so that no node could be orphaned. The simulator does not consider the transmission of data from the cluster head to the sink as this is the same in both methods. The simulator was based on the characterization and performance of an IEEE 802.15.4 transceiver using the Freescale architecture. Xbee radio modules produced by Digi International where characterized in terms of signal strength variability. Radio performance was found to be in agreement with the model of RSSI as a function of distance $[15,16]$ :

$$
R S S I(d)=P_{t} P L\left(d_{0}\right)=10 \eta \log _{10} \frac{d}{d_{0}}+X_{\sigma}
$$

where $P_{t}$ is the transmit power, $P L\left(d_{0}\right)$ is the path loss for a reference distance $d_{0}, \eta$ is the path loss exponent and $X_{\sigma}$ is a Gaussian random noise variable which introduces random variance into the RSSI value. Previous work in $[15,16]$ indicates that this model characterizes the protocol well.

Using the energy model proposed in $\mathrm{LEACH}[1,17]$, the factor impacting the cost of a link is the distance, and the energy dissipation follows the Friss free-space model with power loss given as a function of $d^{2}$. The cost function for transmission is:

$$
E_{T_{x}}(n b, d)=n b * E_{\text {elec }}+n b * E_{a m p} d^{2}
$$

and the cost of reception is

$$
E_{R_{x}}(n b, d)=n b * E_{\text {elec }}
$$

in Joules of energy where $n b$ is the number of bits transmitted, $d$ is the distance, $E_{\text {elec }}$ is the transceiver energy cost in $J / b i t$, and $E_{a m p}$ is the cost for the transceiver to achieve a suitable signal to noise ratio in $J / \mathrm{m}^{2}$.

Thus, the largest energy cost incurred by each node will be the communications with the cluster head during data aggregation. Our method and LEACH can be compared based on the average distance between end nodes and cluster heads. The results are the average of 100 simulation runs.

The average distance between cluster heads and end nodes is on average $11 \%$ lower for RSSI clustering over LEACH as seen in Figure 1. Further, RSSI clustering produces clusters with significantly less variance in the number of cluster heads and average link distance between end node and cluster head. For example, when the goal is 4 cluster heads $(k=4)$, the distribution of the number of cluster heads for the 100 runs of LEACH is in Figure 3. Note the significantly wider range compared to RSSI clustering (Figure 2). Similarly, there is a significantly larger range in average link distance in LEACH (Figure 5) compared to RSSI clustering (Figure 4). Comparing the average link distance histograms in a side by side box plot (Figure 6), for RSSI clustering the range of average link distances is $13.5 \mathrm{~m}$ compared to $38 \mathrm{~m}$ for LEACH. RSSI clustering forms clusters with more uniform size and with a more regular distribution of link lengths across all clusters. For the same number of cluster heads, LEACH produces a less uniform, skewed distribution of longer link lengths over all clusters, with a larger mean value.

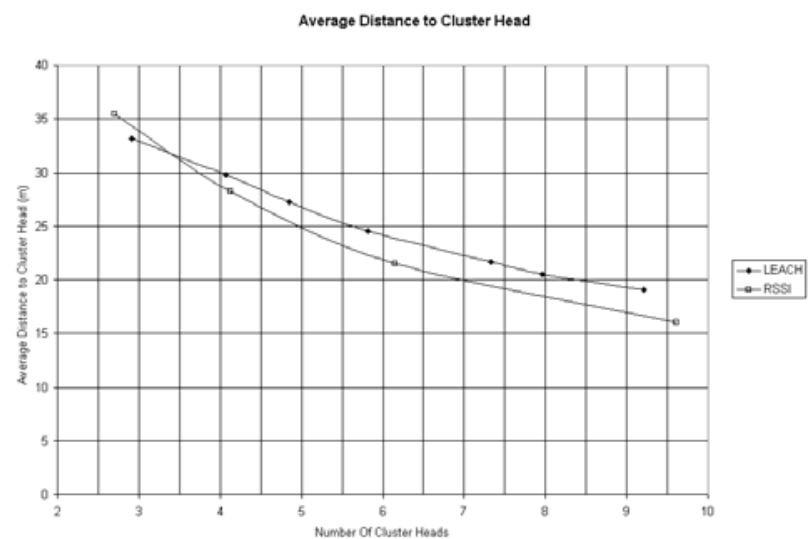

Fig. 1. Average link distance: RSSI vs. LEACH

Since link distance directly relates to energy consumption as given by Equations (3) and (4), RSSI clustering results in less energy usage for transmission and a more even distribution of energy consumption over all nodes. RSSI clustering offers an average of $21 \%$ energy savings in terms of communication costs between end nodes and cluster heads over LEACH due to the lower average link distance and distribution assuming the same communication regime for each protocol after clustering is complete.

RSSI clustering also performs better than LEACH for networks that request a low number of cluster heads. LEACH is not guaranteed to produce a cluster head for each round when a small $k$ is selected. RSSI clustering always produces a cluster head. Table (4) indicates the percentage of rounds without a cluster head as a function of $k$ for LEACH.

In summary, the results show that RSSI clustering per- 
Cluster Head Formation for RSSI Threshold $=0.28(k=4)$

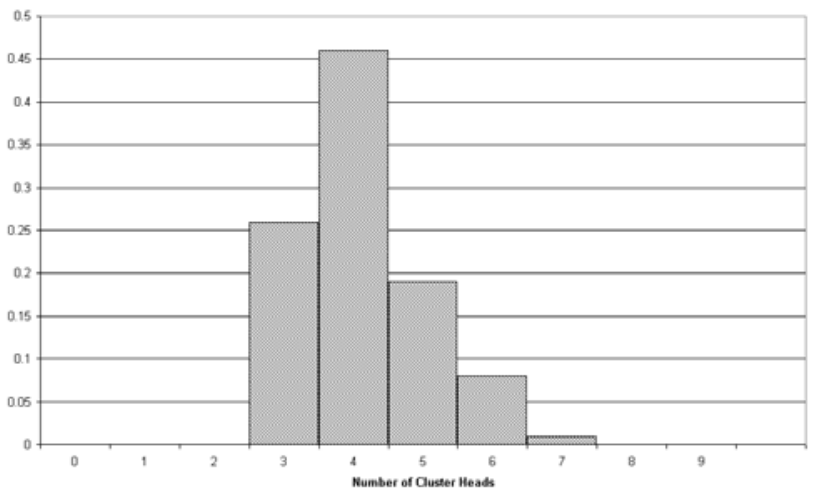

Fig. 2. Distribution of cluster head count for RSSI clustering

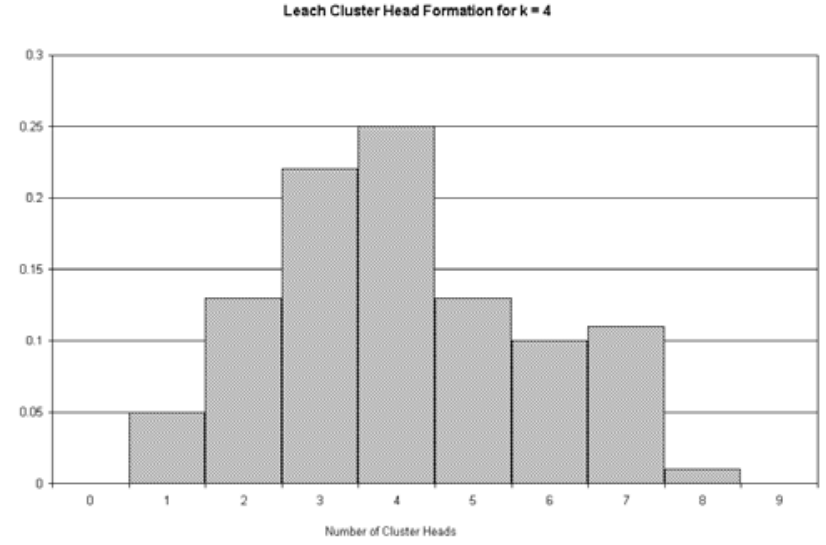

Fig. 3. Distribution of cluster head count for LEACH

forms significantly better than LEACH in constructing uniformly sized clusters with a lower average link distance for the same $k$. The RSSI clustering algorithm results in less energy consumption during data transmission and is supported by hardware implementations of RF signal strength capture following the 802.15.4 standard.

\section{CONCLUSION}

In conclusion, RSSI clustering is a practical solution to the cluster head selection problem. The simulation results empirically show that the RSSI clustering algorithm produces

\begin{tabular}{|c|c|}
\hline$k$ & percentage of rounds without cluster head \\
\hline 1 & $42 \%$ \\
2 & $12 \%$ \\
3 & $6 \%$ \\
\hline
\end{tabular}

Table 1. \% of rounds without a cluster head for LEACH

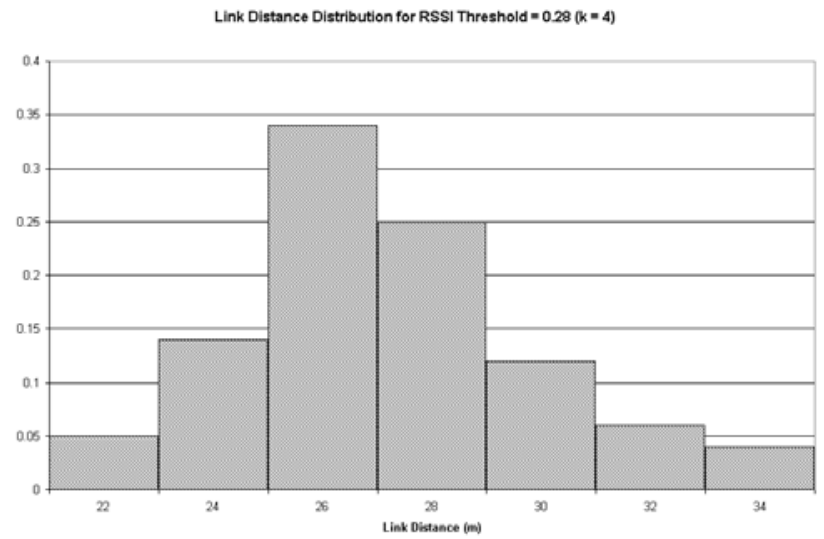

Fig. 4. Distribution of link distances from end nodes to cluster heads for RSSI clustering

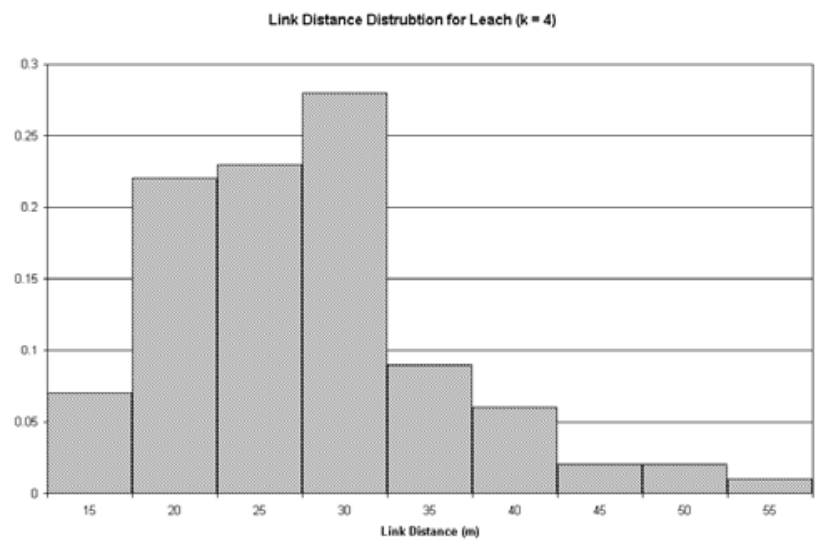

Fig. 5. Distribution of link distances from end nodes to cluster heads for LEACH clustering

more uniformly sized clusters with a lower average end node to cluster head distance which leads to a more uniform energy distribution across the network. RSSI clustering is a more robust algorithm as it is not a probabilistic method. It is adaptable to various configurations and situations, as it makes no assumptions about nodes in the network. Decisions are based on actual parameters and measurements for a given network configuration. Unlike LEACH, which may experience rounds where no cluster head is available, RSSI clustering will always produce a cluster head. This leads to a greater chance of continuous network connectivity.

Future work includes the investigation of dynamic control of $R S S I_{\text {thres }}$ to better improve cluster size and distribution. Work will also be conducted to implement a system wide energy consumption model to better understand the total energy costs for this model in addition to implementing the model in the NS-2 network simulator [18]. 


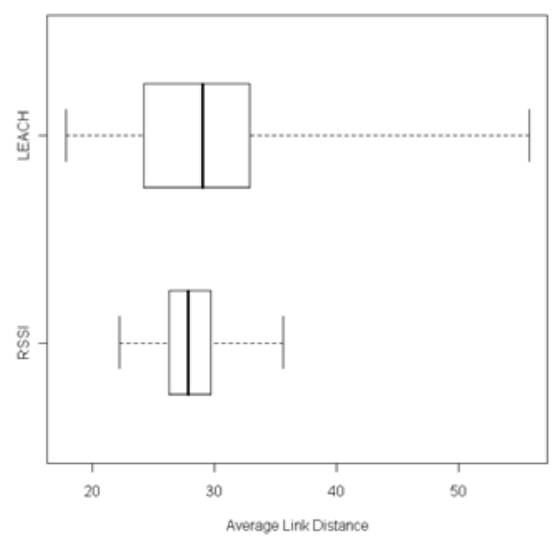

Fig. 6. Side by side box plot comparing average link distances for RSSI and LEACH clustering for $k=4$

\section{REFERENCES}

[1] W. Heinzelman, A. Chandrakasan, and H. Balakrishnan, "An application-specific protocol architecture for wireless microsensor networks," IEEE Transactions on Wireless Communications, vol. 1, no. 4, pp. 660-670, 2002.

[2] A. Amis, R. Prakash, H. Thai, V. Dung, and T. Huynh, "Max-min d-cluster formation in wireless ad hoc networks," in Proceedings of IEEE INFOCOM, 2000, pp. $32-41$.

[3] O. Younis and S. Fahmy, "Distributed clustering in ad hoc sensor networks: A hybrid, energe-efficient approach," IEEE Transactions on Mobile Computing, vol. 3, no. 4, pp. 366-379, 2004.

[4] H. Chan and A. Perrig, "Ace: An emergent algorithm for highly uniform cluster formation," in Proceedings of the First European Workshop on Sensor Networks (EWSN), 2004, pp. 154-171.

[5] R. Khanna, H. Liu, and H. Chen, "Self-organization of sensor networks using genetic algorithms," in IEEE International Conference on Communications, 2006, pp. 3377-3382.

[6] IEEE Computer Society, "IEEE Std.802.15.4-2006," Sept. 2006.

[7] E. Jung, S. Lee, J. Choi, and Y. Park, "A cluster head selection algorithm adopting sensing-awareness and sensor density for wireless sensor networks," IEICE Transactions on Communications, vol. E90.B, no. 9, pp. 2472-2480, 2007.
[8] J.N. Al-Karaki and A.E. Kamal, "Routing techniques in wireless sensor networks: a survey," IEEE Wireless Communications, vol. 11, no. 6, pp. 6-28, Dec 2004.

[9] P. Agarwal and C. Procopiuc, "Exact and approximation algorithms for clustering," in Proceedings of the ninth annual ACM-SIAM symposium on Discrete algorithms, 1998, pp. 658-667.

[10] X. Haiyan and X. Haiying, "A modified cluster head selection algorithm based on random waiting," in IEEE International Conference on Networking, Sensing and Control, 2008, pp. 129-132.

[11] F. Yiming and Y. Jianjun, "The Communication Protocol for Wireless Sensor Network about LEACH," in International Conference on Computational Intelligence and Security Workshops, 2007, pp. 550-553.

[12] F. Xiangning and S. Yulin, "Improvement on LEACH Protocol of Wireless Sensor Network," in International Conference on Sensor Technologies and Applications, 2007, pp. 260-264.

[13] O. Younis, M. Krunz, and S. Ramasubramanian, "Node clustering in wireless sensor networks: recent developments and deployment challenges," IEEE Network, vol. 20, no. 3, pp. 20-25, May-June 2006.

[14] J. Zheng and M. J. Lee, A comprehensive performance study of IEEE 802.15.4, chapter 4, pp. 218-237, IEEE Press, Wiley Interscience, 2006.

[15] D. Lymberopoulos, Q. Lindsey, and A. Savvides, "An Empirical Characterization of Radio Signal Strength Variability in 3-D IEEE 802.15.4 Networks using Monopole Antennas," in Wireless Sensor Networks: Third European Workshop, 2006, pp. 326-341.

[16] R.N. Barbosa, Jd.C. Rodrigues, S.G.C. Fraiha, H.S. Gomes, and G.P.S. Cavalcante, "An empirical model for propagation loss prediction in indoor mobile communications using Pade approximant," in International Conference on Microwave and Optoelectronics, 2005, pp. 625-628.

[17] H. Yang and B. Sikdar, "Optimal Cluster Head Selection in the LEACH Architecture," in IEEE International Performance, Computing, and Communications Conference, 2007, pp. 93-100.

[18] UCB/LBNL/VINT Network Simulator - ns [Online], "NS-2," 2008, Available: http://www.isi.edu/nsnam/ns/. 